\title{
ONE-PARAMETER LEFSCHETZ CLASS OF HOMOTOPIES ON TORUS
}

\author{
WESLEM L. SILVA
}

\begin{abstract}
The main result this paper states that if $F: T \times I \rightarrow T$ is a homotopy on torus then the one-parameter Lefschetz class $L(F)$ of $F$ is given by $L(F)= \pm N(F) \alpha$, where $N(F)$ is the oneparameter Nielsen number of $F$ and $\alpha$ is one of the two generators in $H_{1}\left(\pi_{1}(T), \mathbb{Z}\right)$.
\end{abstract}

\section{INTRODUCTION}

Let $F: T \times I \rightarrow T$ be a homotopy on torus and $G=\pi_{1}\left(T, x_{0}\right)$. R.Geoghegan and A. Nicas in [5] developed an one-parameter theory and defined the one-parameter trace $R(F)$ of $F$. The element $R(F)$ is a 1-chain in $\left.H H_{1}\left(\mathbb{Z} G,(\mathbb{Z} G)^{\phi}\right)\right)$, where the structure of the bimodule $\left.(\mathbb{Z} G)^{\phi}\right)$ is given in section 2. This 1-chain gives information about the fixed points of $F$, that is, using $R(F)$ is possible to define the oneparameter Nielsen number $N(F)$ of $F$ and the one-parameter Lefschetz class $L(F)$ of $F . \mathrm{N}(\mathrm{F})$ is the number of non-zero C-components in $R(F)$ and $L(F)$ is the image of $R(F)$ in $H_{1}(G)$ by homomorfism $\overline{j_{C}}$ : $H_{1}\left(Z\left(g_{C}\right)\right) \rightarrow H_{1}(G)$, induced by inclusion $j_{C}: Z\left(g_{C}\right) \rightarrow G$, where $Z\left(g_{C}\right)$ is the semicentralizer of an element $g_{C}$ which represents the semiconjugacy class $C$. The precise definition is given in [5].

The main purpose this paper is show that for which homotopy on torus then $L(F)= \pm N(F) \alpha$, where $\alpha$ is on of the two generators in $H_{1}(G)$.

In [1] R.B.S.Brooks et al. showed that if $f: X \rightarrow X$ is any map on a k-dimensional torus $X$ then $N(f)=|L(f)|$, where $N(f)$ is the Nielsen

Date: September 11, 2018.

2010 Mathematics Subject Classification. Primary 55M20; Secondary 57Q40, $57 \mathrm{M} 05$.

Key words and phrases. One-parameter fixed point theory, one-parameter Lefschetz class, one-parameter Nielsen number . 
number and $L(f)$ the Lefschetz number of $f$. In some sense our result is a version of this result for one-parameter case when $k=2$.

This paper is organized into five sections, besides this one. In Section 2 contain a review of one-parameter fixed point theory. In section 3 we presented some results of semiconjugacy classes on torus. In Section 4 have the proof of the main result which is the Theorem 4.1.

\section{One-Parameter Fixed Point Theory}

Let $X$ be a finite connected $\mathrm{CW}$ complex and $F: X \times I \rightarrow X$ a cellular homotopy. We consider $I=[0,1]$ with the usual CW structure and orientation of cells, and $X \times I$ with the product $\mathrm{CW}$ structure, where its cells are given the product orientation. Pick a basepoint $(v, 0) \in X \times I$, and a basepath $\tau$ in $X$ from $v$ to $F(v, 0)$. We identify $\pi_{1}(X \times I,(v, 0)) \equiv G$ with $\pi_{1}(X, v)$ via the isomorphism induced by projection $p: X \times I \rightarrow X$. We write $\phi: G \rightarrow G$ for the homomorphism;

$$
\pi_{1}(X \times I,(v, 0)) \stackrel{F_{\#}}{\rightarrow} \pi_{1}(X, F(v, 0)) \stackrel{c_{\mathcal{\tau}}}{\rightarrow} \pi_{1}(X, v)
$$

We choose a lift $\tilde{E}$ in the universal cover, $\tilde{X}$, of $X$ for each cell $E$ and we orient $\tilde{E}$ compatibly with $E$. Let $\tilde{\tau}$ be the lift of the basepath $\tau$ which starts at in the basepoint $\tilde{v} \in \tilde{X}$ and $\tilde{F}: \tilde{X} \times I \rightarrow \tilde{X}$ the unique lift of $F$ satisfying $\tilde{F}(\tilde{v}, 0)=\tilde{\tau}(1)$. We can regard $C_{*}(\tilde{X})$ as a right $\mathbb{Z} G$ chain complex as follows: if $\omega$ is a loop at $v$ which lifts to a path $\tilde{\omega}$ starting at $\tilde{v}$ then $\tilde{E}[\omega]^{-1}=h_{[w]}(\tilde{E})$, where $h_{[\omega]}$ is the covering transformation sending $\tilde{v}$ to $\tilde{\omega}(1)$.

The homotopy $\tilde{F}$ induces a chain homotopy $\tilde{D}_{k}: C_{k}(\tilde{X}) \rightarrow C_{k+1}(\tilde{X})$ given by $\tilde{D}_{k}(\tilde{E})=(-1)^{k+1} F_{k}(\tilde{E} \times I) \in C_{k+1}(\tilde{X})$, for each cell $\tilde{E} \in \tilde{X}$. This chain homotopy satisfies; $\tilde{D}(\tilde{E} g)=\tilde{D}(\tilde{E}) \phi(g)$ and the boundary operator $\tilde{\partial}_{k}: C_{k}(\tilde{X}) \rightarrow C_{k-1}(\tilde{X})$ satisfies; $\tilde{\partial}(\tilde{E} g)=\tilde{\partial}(\tilde{E}) g$.

Define endomorphism of, $\oplus_{k} C_{k}(\tilde{X})$, by $\tilde{D}_{*}=\oplus_{k}(-1)^{k+1} \tilde{D}_{k}, \tilde{\partial}_{*}=$ $\oplus_{k} \tilde{\partial_{k}}, \tilde{F_{0 *}}=\oplus_{k}(-1)^{k} \tilde{F_{0 k}}$ and $\tilde{F_{1 *}}=\oplus_{k}(-1)^{k} \tilde{F_{1 k}}$. We consider trace $\left(\tilde{\partial}_{*} \otimes\right.$ $\left.\tilde{D}_{*}\right) \in H H_{1}\left(\mathbb{Z} G,(\mathbb{Z} G)^{\phi}\right)$. This is a Hochschild 1-chain whose boundary is: $\operatorname{trace}\left(\tilde{D}_{*} \phi\left(\tilde{\partial}_{*}\right)-\tilde{\partial}_{*} \tilde{D}_{*}\right)$. We denote by $G_{\phi}(\partial(F))$ the subset of $G_{\phi}$ consisting of semiconjugacy classes associated to fixed points of $F_{0}$ or $F_{1}$. 
Definition 2.1. The one-parameter trace of homotopy $F$ is:

$$
\begin{gathered}
R(F) \equiv T_{1}\left(\tilde{\partial}_{*} \otimes \tilde{D}_{*} ; G_{\phi}(\partial(F))\right) \in \bigoplus_{C \in G_{\phi}-G_{\phi}(\partial(F))} H H_{1}\left(\mathbb{Z} G,(\mathbb{Z} G)^{\phi}\right)_{C} \\
\cong \bigoplus_{C \in G_{\phi}-G_{\phi}(\partial(F))} H_{1}\left(Z\left(g_{C}\right)\right) .
\end{gathered}
$$

Definition 2.2. The $C$-component of $R(F)$ is denoted by $i(F, C) \in$ $H H_{1}\left(\mathbb{Z} G,(\mathbb{Z} G)^{\phi}\right)_{C}$. We call it the fixed point index of $F$ corresponding to semiconjugacy class $C \in G_{\phi}$. A fixed point index $i(F, C)$ of $F$ is zero if the all cycle in $i(F, C)$ is homologous to zero.

Definition 2.3. Given a cellular homotopy $F: X \times I \rightarrow X$ the oneparameter Nielsen number, $N(F)$, of $F$ is the number of nonzero fixed point indices.

Definition 2.4. The one-parameter Lefschetz class, $L(F)$, of $F$ is defined by;

$$
L(F)=\sum_{C \in G_{\phi}-G_{\phi}(\partial F)} j_{C}(i(F, C))
$$

where $j_{C}: H_{1}\left(Z\left(g_{C}\right)\right) \rightarrow H_{1}(G)$ is induced by the inclusion $Z\left(g_{C}\right) \subset G$.

From [5] we have the following theorems.

Theorem 2.1 (Invariance). Let $F, G: X \times I \rightarrow X$ be cellular; if $F$ is homotopic to $G$ relative to $X \times\{0,1\}$ then $R(F)=R(G)$.

Theorem 2.2 (one-parameter Lefschetz fixed point theorem). If $L(F) \neq$ 0 then every map homotopic to $F$ relative to $X \times\{0,1\}$ has a fixed point not in the same fixed point class as any fixed point in $X \times\{0,1\}$. In particular, if $F_{0}$ and $F_{1}$ are fixed point free, every map homotopic to $F$ relative to $X \times\{0,1\}$ has a fixed point.

Theorem 2.3 (one-parameter Nielsen fixed point theorem). Every map homotopic to $F$ relative to $X \times\{0,1\}$ has at least $N(F)$ fixed point classes other than the fixed point classes which meet $X \times\{0,1\}$. In particular, if $F_{0}$ and $F_{1}$ are fixed point free maps, then every map homotopic to $F$ relative to $X \times\{0,1\}$ has at least $N(F)$ path components.

For a complete description of the one-parameter fixed point theory see [5]. 


\section{Semiconjugacy Classes on torus}

In this subsection we describe some results about the semiconjugacy classes in the torus related to a homotopy $F: T \times I \rightarrow T$. We will consider the homomorphism $\phi=c_{\tau} \circ F_{\#}$ given above.

We take $w=[(0,0)] \in T$ and $G=\pi_{1}(T, w)=\left\{u, v \mid u v u^{-1} v^{-1}=1\right\}$, where $u \equiv a$ and $v \equiv b$. Thus, given the homomorphism $\phi: G \rightarrow$ $G$ we have $\phi(u)=u^{b_{1}} v^{b_{2}}$ and $\phi(v)=u^{b_{3}} v^{b_{4}}$. Therefore, $\phi\left(u^{m} v^{n}\right)=$ $u^{m b_{1}+n b_{3}} v^{m b_{2}+n b_{4}}$, for all $m, n \in \mathbb{Z}$. We denote this homomorphism by the matrix:

$$
[\phi]=\left(\begin{array}{ll}
b_{1} & b_{3} \\
b_{2} & b_{4}
\end{array}\right)
$$

Proposition 3.1. Two elements $g_{1}=u^{m_{1}} v^{n_{1}}$ and $g_{2}=u^{m_{2}} v^{n_{2}}$ in $G$ belong to the same semiconjugacy class, if and only if there are integers $m, n$ satisfying the following equations:

$$
\left\{\begin{array}{c}
m\left(b_{1}-1\right)+n b_{3}=m_{2}-m_{1} \\
m b_{2}+n\left(b_{4}-1\right)=n_{2}-n_{1}
\end{array}\right.
$$

Proof. If there is $g=u^{m} v^{n} \in G$ satisfying $g_{1}=g g_{2} \phi(g)^{-1}$ then we obtain the equation of the proposition. The other direction is analogous.

We take the isomorphism $\Theta: G \rightarrow \mathbb{Z} \times \mathbb{Z}$ such that $\Theta\left(u^{m} v^{n}\right)=$ $(m, n)$. By Proposition 3.1 two elements $g_{1}=u^{m_{1}} v^{n_{1}}$ and $g_{2}=u^{m_{2}} v^{n_{2}}$ in $G$ belong to the same semiconjugacy class, if and only if there is $z \in \mathbb{Z} \times \mathbb{Z}$ satisfying: $([\phi]-I) z=\Theta\left(g_{2} g_{1}^{-1}\right)$, where $I$ is the identity matrix. If determinant of the matrix $([\phi]-I)$ is zero then will have an infinite amount of elements in a semiconjugacy class.

Corollary 3.2. For each $g \in G$ the semicentralizer $Z(g)$ is isomorphic to the kernel of $[\phi]-I$.

Lemma 3.1. The 1-chain, $u^{k} v^{l} \otimes u^{m} v^{n}$, is a cycle if and only if the element $(k, l) \in \mathbb{Z} \times \mathbb{Z}$ belongs to the kernel of $[\phi]-I$.

Proof. If $u^{k} v^{l} \otimes u^{m} v^{n}$ is a cycle, then $0=d_{1}\left(u^{k} v^{l} \otimes u^{m} v^{n}\right)=u^{m} v^{n} \phi\left(u^{k} v^{l}\right)-$ $u^{k} v^{l} u^{m} v^{n}=u^{m} v^{n} u^{k b_{1}+l b_{3}} v^{k b_{2}+l b_{4}}-u^{k} v^{l} u^{m} v^{n}=u^{m+k b_{1}+l b_{3}} v^{k b_{2}+l b_{4}+n}-$ $u^{k+m} v^{l+n}$. This implies $k\left(b_{1}-1\right)+l b_{3}=0$ and $k b_{2}+l\left(b_{4}-1\right)=0$. The other direction is analogous. 
Corollary 3.3. If the matrix of the homomorphism $\phi$ is given by

$$
[\phi]=\left(\begin{array}{ll}
1 & b_{3} \\
0 & b_{4}
\end{array}\right)
$$

with $b_{3} \neq 0$ or $b_{4} \neq 1$, then the 1-chain, $u^{k} v^{l} \otimes u^{m} v^{n}$, is a cycle if and only if $l=0$.

By definition given a 2-chain $u^{s} v^{t} \otimes u^{k} v^{l} \otimes u^{m} v^{n} \in C_{2}\left(\mathbb{Z} G,(\mathbb{Z} G)^{\phi}\right)_{C}$ then

$$
\begin{aligned}
& d_{2}\left(u^{s} v^{t} \otimes u^{k} v^{l} \otimes u^{m} v^{n}\right)=u^{k} v^{l} \otimes u^{m+s b_{1}+t b_{3}} v^{n+s b_{2}+t b_{4}}-u^{k+s} v^{l+t} \otimes u^{m} v^{n} \\
& +u^{s} v^{t} \otimes u^{k+m} v^{l+n} .
\end{aligned}
$$

Proposition 3.4. The 1-chain, $u^{k} \otimes u^{m} v^{n} \in C_{1}\left(\mathbb{Z} G,(\mathbb{Z} G)^{\phi}\right)_{C}$, is homologous to the 1-chain, $k u \otimes u^{m+k-1} v^{n}$, for all $k, m, n \in \mathbb{Z}$.

Proof. Note that for $k=0$ and 1 the proposition is true. We suppose that for some $s>0 \in \mathbb{Z}$, the 1-chain $u^{s} \otimes u^{m} v^{n}$ is homologous to the 1-chain $s u \otimes u^{m+s-1} v^{n}$, we will write $u^{s} \otimes u^{m} v^{n} \sim s u \otimes u^{m+s-1} v^{n}$. Considering to the 2-chain $u^{s} \otimes u \otimes u^{m} v^{n} \in C_{2}\left(\mathbb{Z} G,(\mathbb{Z} G)^{\phi}\right)$ we have

$$
\begin{aligned}
d_{2}\left(u^{s} \otimes u \otimes u^{m} v^{n}\right) & =u \otimes u^{m+s} v^{n}-u^{s+1} \otimes u^{m} v^{n}+u^{s} \otimes u^{1+m} v^{n} \\
& \sim u \otimes u^{m+s} v^{n}-u^{s+1} \otimes u^{m} v^{n}+s u \otimes u^{1+m+s-1} v^{n} \\
& =(s+1) u \otimes u^{m+(s+1)-1} v^{n}-u^{s+1} \otimes u^{m} v^{n} .
\end{aligned}
$$

Therefore $(s+1) u \otimes u^{m+(s+1)-1} v^{n} \sim u^{s+1} \otimes u^{m} v^{n}$. Using induction, we obtain the result. The case in which $k<0$ is analogous.

The proof of following results can be found in [8].

Proposition 3.5. In the case $b_{1}=1$ and $b_{2}=0$ each 1-cycle $\sum_{i=1}^{t} a_{i} u^{k_{i}} v^{l_{i}} \otimes$ $u^{m_{i}} v^{n_{i}} \in C\left(\mathbb{Z} G,(\mathbb{Z} G)^{\phi}\right)$ is homologous to a 1-cycle the following form: $\sum_{i=1}^{\bar{t}} \bar{a}_{i} u \otimes u^{\bar{m}_{i}} v^{\bar{n}_{i}}$.

Proposition 3.6. Each 1-cycle $u \otimes u^{m} v^{n} \in H H_{1}\left(\mathbb{Z} G,(\mathbb{Z} G)^{\phi}\right)_{C}$ is not trivial, that is, is not homologous to zero. 
Corollary 3.7. Let $\sum_{i=1}^{t} u \otimes u^{m_{i}} v^{n_{i}} \in H H_{1}\left(\mathbb{Z} G,(\mathbb{Z} G)^{\phi}\right), m_{i}, n_{i} \in \mathbb{Z}$ be a cycle. If the cycles $u \otimes u^{m_{i}} v^{n_{i}}$ and $u \otimes u^{m_{j}} v^{n_{j}}$ are in different semiconjugacy classes for $i \neq j, i, j \in\{1, \ldots, t\}$, then $\sum_{i=1}^{t} u \otimes u^{m_{i}} v^{n_{i}} i s$ a nontrivial cycle. Each cycle $u \otimes u^{m_{i}} v^{n_{i}}$ projects to the class $[u]$ that is one of the two generators of $H_{1}(G)$.

\section{Homotopies on torus}

Let $F: T \times I \rightarrow T$ be a homotopy on torus $T$.

Proposition 4.1. Let $F: T \times I \rightarrow T$ be a homotopy. Suppose that $L\left(F_{t}\right)=0$ for each $t \in I$. Then $F$ is homotopic to a homotopy $H$ with $H$ transverse the projection $P: T \times I \rightarrow T$ such that Fix $\left(H_{\mid T \times\{0,1\}}\right)=$ ptyset.

Proof. We can choose a homotopy $F_{0}$ homotopic to $F$ with $F_{0}$ transverse the projection $P$. Therefore, $F i x\left(F_{0}\right)$ is transverse, that is, $\operatorname{Fix}\left(F_{0}\right) \cap(T \times\{t\})$ is finite. Since $L\left(F_{\mid T}\right)=L\left(F_{0 \mid T}\right)=0$ then for each $\frac{1}{2}>\epsilon>0$ we can deform $F_{0}$ to a homotopy $F_{1}$ such that $F_{1}(x, t)=F_{0}(x, t)$ for each $(x, t) \in T \times[\epsilon, 1-\epsilon]$ and $F_{1}$ has no fixed points in $T \times\{0,1\}$. In fact, take $A: T \times I \times I \rightarrow T$ defined by

$$
A((x, y), t, s)= \begin{cases}F_{0}(x, y, 0) & \text { if } 0 \leq t \leq s \epsilon \\ F_{0}\left(x, y, \frac{1}{1-2 s \epsilon}(t-s \epsilon)\right) & \text { if } s \epsilon \leq t \leq 1-s \epsilon \\ F_{0}(x, y, 1) & \text { if } 1-s \epsilon \leq t \leq 1\end{cases}
$$

Since $L\left(F_{0 \mid T}\right)=0$, there are two homotopies $H_{1}, H_{2}: T \times I \rightarrow T$ such that $H_{1}(x, y, 1)=F_{0}(x, y, 0), H_{2}(x, y, 0)=F_{0}(x, y, 1)$ and $H_{1}(x, y, 0)$, $H_{2}(x, y, 1)$ are fixed points free maps. Considere the homotopy $B$ : $T \times I \times I \rightarrow T$ defined by;

$$
B((x, y), t, s)= \begin{cases}H_{1}\left(x, y, \frac{t}{\epsilon} s\right) & \text { if } 0 \leq t \leq \epsilon \\ F_{0}\left(x, y, \frac{1}{1-2 \epsilon}(t-\epsilon)\right) & \text { if } \epsilon \leq t \leq 1-\epsilon \\ H_{2}\left(x, y, \frac{(t-1+\epsilon)}{\epsilon} s\right) & \text { if } 1-\epsilon \leq t \leq 1\end{cases}
$$

Thus, taking

$$
J((x, y), t, s)= \begin{cases}A((x, y), t, 2 s) & \text { if } \quad 0 \leq s \leq \frac{1}{2} \\ B((x, y), t), 2 s-1) & \text { if } \quad \frac{1}{2} \leq s \leq 1\end{cases}
$$


we have a homotopy between $F_{0}$ and a map $H$ where $H$ satisfying the hypothesis of the theorem. Note that we can choose $\frac{1}{2}>\epsilon>0$ such that $F i x\left(F_{0}\right) \subset T \times[\epsilon, 1-\epsilon]$ because $F i x\left(F_{0}\right)$ is contained in $\operatorname{int}(T \times I)$. Thus, Fix $(H)$ is transverse.

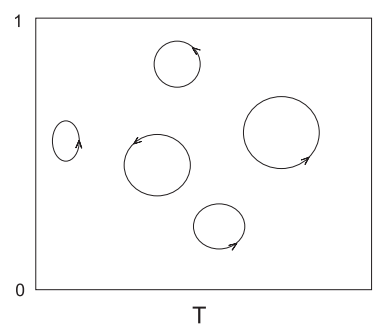

Figure 1. Circles in Fix $(F)$.

Let Fix $(F, \partial)$ be the subset of Fix $(F)$ consisting of those circles of fixed points which are not in the same fixed point class as any fixed point of $F_{0}$ or $F_{1}$. From [5] Fix $(F)$ consists of oriented arcs and circles.

From Proposition 4.1 if $F: T \times I \rightarrow T$ is a homotopy and $P$ : $T \times I \rightarrow T$ the projection then we can choose $F$ such that $F i x(F)$ is transverse the projection $P$. Thus, Fix $(F, \partial)$ is a closed oriented 1manifold in the interior of $T \times I \times T$. Let $E_{F}$ be space of all paths $\omega(t)$ in $T \times I \times T$ from the graph $\Gamma_{F}=\{(x, t, F(x, t)) \mid(x, t) \in T \times I\}$ of $F$ to the graph $\Gamma_{P}=\{(x, t, x) \mid(x, t) \in T \times I\}$ of $P$ with the compact-open topology, that is, maps $\omega:[0,1] \rightarrow T \times I \times T$ such that $\omega(0) \in \Gamma(F)$ and $\omega(1) \in \Gamma(P)$.

Let $C_{1}, \ldots, C_{k}$ be isolated circles in Fix $(F) \cap \operatorname{int}(T \times I)$, oriented by the natural orientations, and $V=\bigcup C_{j}$. Then $V$ determines a family of circles $V^{\prime}$ in $E_{F}$ via constant paths, i.e. each oriented isolated circle of fixed points $C: S^{1} \rightarrow T \times I$ of $F$ determines an oriented circle $C^{\prime}: S^{1} \rightarrow E$ defined by $\operatorname{con}(C(z))$ where $\operatorname{con}(C(z)$ is the constant path at $C(z)=\left(x, t_{0}\right)$, that is, $\operatorname{con}(C(z))(t)=\left(x, t_{0}, x\right)$ for each $t \in$ $[0,1]$. Therefore, we can write $\sum i\left(F, C_{j}\right)$. $\left[C_{j}^{\prime}\right] \in H_{1}\left(E_{F}\right)$. Since $C_{j}$ is transverse then $i\left(F, C_{j}\right)=1$ for all $j$, see [2]. From [5] we have;

Proposition 4.2. Since $\pi_{2}(T)=0$ then there is a isomorphism $\Psi$ : $H_{1}\left(E_{F}\right) \rightarrow H H_{1}\left(\mathbb{Z} G,(\mathbb{Z} G)^{\phi}\right)$, where $G=\pi_{1}\left(T, x_{0}\right)$. 
Remarks 4.1. From [2], section $I V$, given $F: T \times I \rightarrow T$ a homotopy then we can to deform $F$ to a homotopy $G$ such that in each fixed point class of $G$ has an unique circle, and this circle is transverse the projection.

Now we are going to proof the main result.

Theorem 4.1 (Main Theorem). If $F: T \times I \rightarrow T$ is a homotopy then the one-parameter Lefschetz class $L(F)$ of $F$ satisfies $L(F)= \pm N(F) \alpha$ where $\alpha$ is one of the two generators of $H_{1}\left(\pi_{1}(T), \mathbb{Z}\right)$.

Proof. The proof this theorem will be done in two cases. Case I when $\operatorname{det}([\phi]-I)=L\left(F_{\mid T}\right)=0$ and case II when $\operatorname{det}([\phi]-I)=L\left(F_{\mid T}\right) \neq 0$.

\section{Case I}

Let us suppose that the homomorphism $\phi$ is induced by a homotopy $F$ satisfies $\operatorname{det}([\phi]-I)=0$. Using the notation above we can suppose which $\phi$ is given by

$$
[\phi]=\left(\begin{array}{ll}
1 & b_{3} \\
0 & b_{4}
\end{array}\right),
$$

and $[\phi] \neq I \equiv$ (Identity), that is, $b_{1}=1$ and $b_{2}=0$, with $b_{3} \neq 0$ or $b_{4}-1 \neq 0$. This is done choosing a base $\{v, w\}$ for $T=\mathbb{R}^{2} / \mathbb{Z}^{2}$, where $v$ is a eigenvector of $[\phi]$ associated to 1 .

Note that if $[\phi]=I$ then $R(F)=0$ because any $F$ can be deformed to a fixed point free map. For example, take the homotopy $F: T \times I \rightarrow T$ defined by;

$$
F((x, y), t)=\left(x+c_{1} t+\epsilon, y+c_{2} t\right)
$$

with $\epsilon$ any irrational number between 0 and 1 . We will have $\left[F_{\#}\right]=$ $[\phi]=I$, but $F$ is a fixed point free map. Thus $R(F)=0$, which implies $L(F)=N(F)=0$. Therefore, henceforth we suppose $[\phi] \neq I$.

Since $T$ is a polyhedron then $T$ is a regular $\mathrm{CW}$-complex. Thus, for any cellular decomposition of the torus the entries of matrices of the operators $\tilde{\partial}_{1}$ and $\tilde{\partial}_{2}$ will be composed by elements $0, \pm 1, \pm u, \pm v$, because the incindence number of a 2 -cell in a 1-cell is \pm 1 and the the incindence of one 1 -cell in one 0 -cell is \pm 1 , see chapter II of [10]. 
Therefore chosen an orientation to each cell in a decomposition cellular to the torus then the one-parameter trace $R(F)$ will be the form of the following matrix:

$$
R(F)=\operatorname{tr}\left(\begin{array}{cc}
{\left[-\tilde{\partial}_{1}\right] \otimes\left[\tilde{D}_{0}\right]} & 0 \\
0 & {\left[\tilde{\partial}_{2}\right] \otimes\left[\tilde{D}_{1}\right]}
\end{array}\right)
$$

where $\left[\tilde{\partial}_{1}\right]_{i j},\left[\tilde{\partial}_{2}\right]_{k l} \in\{0, \pm 1, \pm u, \pm v$,$\} . Thus, we can write$

$$
R(F)= \pm 1 \otimes\left(\sum_{i=1}^{m} g_{i}\right)+u \otimes\left(\sum_{j=1}^{n} h_{j}\right)+v \otimes\left(\sum_{k=1}^{p} t_{k}\right)
$$

or only $-u$ or $-v$, where $g_{i}=u^{m_{i}} v^{n_{i}}, h_{j}=u^{x_{j}} v^{y_{j}}$ and $t_{k}=u^{z_{k}} v^{w_{k}}$. We will suppose which $R(F)$ is write like above. The case with $-u$ or $-v$ the proof is analogous.

From Lemma 4.1 of [8] the element $\pm 1 \otimes\left(\sum_{i}^{m} g_{i}\right)$ is homologous to zero. By Proposition 4.1 we can suppose that $F$ has no fixed points in $T \times\{0,1\}$. In this situation $R(F)$ will be a 1 -cycle in $H H_{1}\left(\mathbb{Z} G,(\mathbb{Z} G)^{\phi}\right)$. Thus, By Proposition 3.5, the sum $v \otimes\left(\sum_{k}^{p} t_{k}\right)$ can not be appear in one-parameter trace $R(F)$ of $F$. Therefore, in this case the trace $R(F)$ has the form:

$$
R(F)= \pm 1 \otimes\left(\sum_{i=1}^{m} g_{i}\right)+u \otimes\left(\sum_{j=1}^{n} h_{j}\right)
$$

From Proposition 4.2 each C-component nonzero in $R(F)$ will represent by one unique cycle. Therefore the one-parameter Nielsen number in this case will be $N(F)=n$.

From section 2 the one-parameter Lefschetz class is the image of $R(F)$ in $H_{1}(G)$ by induced of inclusion $i: Z\left(g_{C}\right) \rightarrow G$. Thus, each element $u \otimes h_{j}$ is sending in $H_{1}(G)$ in the class $[u]$, that is, the image of $R(F)$ in $H_{1}(G)$ will be

$$
L(F)=\sum_{j=1}^{n}[u]=n[u]=N(F)[u]
$$

Take $\alpha=[u]$, which is one of the two generators of $H_{1}(G)$. If we consider left action instead right action in the covering space we will 
obtain $L(F)=-N(F)[u]$. Therefore,

$$
L(F)= \pm N(F) \alpha
$$

\section{Case II}

In this case we have $\operatorname{det}([\phi]-I)=L\left(F_{\mid T}\right) \neq 0$. Therefore, by Corollary 3.2, for each element $g \in G$ the semicentralizer, $Z(g)$, of $g$ in $G$ is trivial. Thus, $H_{1}\left(Z\left(g_{C}\right)\right)=0$ for each semiconjugacy class $C$, that is, $H H_{1}\left(\mathbb{Z} G,(\mathbb{Z} G)^{\phi}\right)=0$ which implies $R(F)=0$. In this case we have $L(F)=N(F)=0$.

We have other interpretation in Case II. Note that by definition of $R(F)$ in section 2 we are not considering in trace $R(F)$ the semiconjugacy classes represented by fixed point classes which meet $T \times\{0,1\}$. If we consider all fixed points classes then the trace $R(F)$ has the form:

$$
R(F)= \pm 1 \otimes\left(\sum_{i=1}^{m} g_{i}\right)+v \otimes\left(\sum_{k=1}^{p} t_{k}\right.
$$

because in this situation can not be appear circles in Fix $(F)$, but only arcs join $T \times\{0\}$ to $T \times\{1\}$. By Proposition $3.5 R(F)$ can not be a 1-cycle. Since for each $t$ the map $F_{t}$ can be deformed to a map with $L\left(F_{\mid T}\right)$ fixed points, then from Theorem 3.3 of [4] we will have $p=L\left(F_{\mid T}\right)=\operatorname{det}([\phi]-I)$, i.e. in this case $F i x(F)$ will be compose by $\operatorname{det}([\phi]-I)=L\left(F_{\mid T}\right)$ arcs join $T \times\{0\}$ to $T \times\{1\}$.

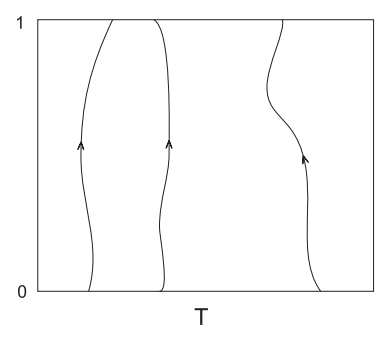

Figure 2. Arcs in Fix $(F)$. 


\section{REFERENCES}

1. R.B.S.Brooks, R.F.Brown, J.Park and D.H.Taylor, Nielsen numbers of maps of tori, Proc. Amer. Math. Soc., vol. 52, 1975.

2. D. Dimovski, One-parameter fixed point indices, Pacific Journal of Math. 2, vol. $164,1994$.

3. D. Dimovski and R. Geoghegan, One-parameter fixed point theory, Forum Math. 2, 1990, 125-154.

4. D.L.Gonçalves, M.R.Kelly, Maps into the torus and minimal coincidence sets for homotopies, Fund. Math., vol. 172, 2002.

5. R. Geoghegan and A. Nicas, Parametrized Lefschetz-Nielsen fixed point theory and Hochschild homology traces, Amer. J. Math. 116, 1994, 397-446.

6. R. Geoghegan and A. Nicas, Trace and torsion in the theory of flows, Topology, vol. 33, No. 4, 1994, 683-719.

7. R. Geoghegan, A. Nicas and D. Schütz, Obstructions to homotopy invariance in parametrized fixed point theory, Geometry and Topology: Aarhus, Contemp. Math. vol. 258, 2000, 351-369.

8. W. L. Silva, Minimal fixed point set of fiber-preserving maps on T-bundles over $S^{1}$, Topology and its Applications, 173, 2014, 240-263.

9. H. Schirmer, Fixed points sets of homotopies, Pacific J. Math. 108, 1983, $191-202$.

10. G. W. Whitehead, Elements of Homotopy Theory, Springer-Verlag, 1918.

(Weslem Liberato Silva) Departamento de Ciências Exatas e Tecnológicas (DCET), Universidade Estadual de Santa Cruz (UESC), Ilhéus - BA, 2015

E-mail address: wlsilva@uesc.br 